\title{
Maiores Empresas Brasileiras no Período 2003-2010: Análise sob à luz da Lei de Zipf
}

\author{
Wellington Ribeiro Justo \\ Professor do Curso de Economia da Universidade Regional do Cariri (URCA). \\ Endereço para contato: Rua Nelson Alencar, 490 - Crato - CE \\ CEP: 63100-060 - E-mail: justowr@yahoo.com.br
}

\section{Katsuk Rodrigues Souza}

Economista e Especialista em Administração Financeira pela Universidade Regional do Cariri (URCA). Endereço para contato: Rua do Limoeiro, 89 - Bairro São Miguel - Juazeiro do Norte - CE CEP: 63020-070 - E-mail: kat_krs@hotmail.com

Recebido em 18 de novembro de 2014. Aceito em 12 de janeiro de 2015.

\section{Resumo}

Em determinadas situações a teoria não pode ser testada empiricamente seja pela falta de formalização matemática, falta de modelos empíricos ou mesmo pela falta de dados. Por outro lado tem uma regularidade empírica que carecia de uma teoria para dar sustentação que é a lei da ordem de tamanho. Recentemente teorias em diversas áreas passaram a dar suporte a esta regularidade empírica sendo uma delas no campo da organização industrial. Assim, este estudo explora esta literatura e testa a Lei de Zipf na distribuição do ranking das maiores empresas brasileiras para o período 2003 a 2010 com dados em painel. Os resultados sugerem que a hipótese da Lei de Zipf é aceita apenas quando o número de empresas na amostra é pequeno. À medida que o número de empresas aumenta os resultados apontam que as menores empresas crescem mais rapidamente que as maiores indicando convergência no tamanho.

\section{Palavras-chave}

Lei de Zipf, Dados em painel; Organização Industrial.

\begin{abstract}
In certain situations, the theory cannot be empirically tested due to the lack of mathematical formalism, lack of empirical or even the lack of data models. Moreover an empirical regularity that has lacked a theory to support that is the law of order size. Recently theories in many areas began to support this empirical regularity one being in the field of industrial organization. This study explores this literature and tests the Zipf's Law distribution of the ranking of the largest Brazilian companies for the period 2003-2010 with panel data. The results suggest that the hypothesis of Zipf's Law is only accepted when the number of firms in the sample is small. As the number of firms increases the results indicate that smaller firms grow faster than larger indicating convergence in size of them.
\end{abstract}

\section{Keywords}

Zipf's Law, Panel data; Industrial Organization. 


\section{Introdução}

Há teorias que são de difícil aplicação empírica, seja pela falta de formalização matemática dos problemas que impede a utilização de modelos aplicados, seja pela falta de dados. Por outro lado há uma regularidade empírica, a lei da ordem do tamanho que carecia de uma fundamentação teórica. A partir da formulação de teoria em vários campos da ciência para esta regularidade empírica, várias foram as suas aplicações.

Este trabalho faz uso desta inovação e parte para a aplicação na distribuição do ranking das maiores empresas brasileiras no período entre 2003 e 2010. Este período compreende os dois mandatos do Presidente Luiz Inácio da Silva (LULA).

O governo Lula teve como um dos pilares da sua política econômica a elevação do consumo das famílias que refletiu nas estratégias comerciais e, por conseguinte no faturamento das empresas brasileiras. Também, formulou uma Política Industrial, Tecnológica e de Comércio Exterior (PITCE), que de acordo com Cano e Silva (2010) tratase de um conjunto de medidas distribuídas em programas de política industrial, cujos objetivos eram aumentar a eficiência da estrutura produtiva, elevar a capacidade de inovação das empresas brasileiras, e expandir as exportações.

Porém no segundo mandato, houve uma mudança no foco da política econômica. Principalmente após a crise de 2008, que para evitar uma recessão na economia brasileira teve que priorizar o mercado interno, tomando inúmeras medidas, das quais uma delas foi a redução dos impostos, afetando diretamente as indústrias, na tentativa de amenizar os efeitos da referida crise.

A queda na produção da indústria de transformação e nas exportações de manufaturados só não foi maior graças ao socorro financeiro que o governo prestou a vários setores e à redução temporária de impostos indiretos na crise de 2008 (CANO, 2009).

Outra medida criada no governo Lula foi um novo programa de promover a Política de Desenvolvimento Produtivo (PDP) cujo objetivo é a sustentação de um longo ciclo de desenvolvimento produtivo, apoiado no investimento, na inovação, na competitividade das empresas e na ampliação das exportações (CANO e SILVA, 2010).

Estas medidas por não alcançarem todos os setores e nem todas as empresas dos setores beneficiados pode ter afetado a competitividade destes mercados e possivelmente trazendo modificações no ranking das maiores empresas brasileiras.

Desta forma, o objetivo principal deste artigo é testar a aplicabilidade da Lei de Zipf para as maiores empresas brasileiras, no período 2003 a 2010 através da estimação com dados em painel.

A aplicação da Lei de Zipf no caso brasileiro se dá em vários campos, como por exemplo, Por Justo (2012) e Justo (2014), para verificar a distribuição do tamanho das cidades brasileiras. Desta forma, este trabalho avança na literatura por fazer uso da relativamente recente formalização de uma teoria que justifica a aplicação desta regularidade empírica à distribuição das empresas em uma economia.

O artigo está estruturado da seguinte forma: além da introdução traz na segunda seção o referencial teórico. A terceira seção traz a metodologia. A seção seguinte contemplam os resultados e finalmente a última seção são apresentadas as conclusões. 


\section{Referencial Teórico}

Para compreender a regra de ordem de tamanho das empresas bem como seu surgimento e aplicação na organização industrial, é necessário explanar a organização industrial e as inovações tecnológicas que provocam competitividade no mercado, da qual será visto a seguir.

\subsection{A Organização Industrial}

Nesta seção busca-se respaldado na teoria para evidenciar que o tamanho das firmas é importante para influenciar o comportamento do mercado e como a organização industrial trata a elevação dos ganhos de competitividade.

Schumpeter tenta incorporar o progresso técnico como variável-chave do processo de evolução da firma e do mercado. Assim os pensamentos neo-schumpeterianos vêm da biologia evolucionista que lançam bases para a construção da teoria da firma. Os elementos como aprendizagem, rotinas, regimes tecnológicos, organização e competência, capacitações dinâmicas, entre outros são fundamentais na difusão conceitual da firma (CAMPOS e PAULA, 2006).

Para Schumpeter as mudanças criativas na combinação de fatores produtivos iniciados pelo empreendedor estão interligadas através do processo de mudança institucional. Com base em ferramentas analíticas é possível identificar três principais modos de tais ligações e as alterações correspondentes: imersão, complementaridades, e agrupamento. Os dois primeiros modos tendem a proporcionar o processo de mudança institucional com características, mais ou menos marginais dependentes do caminho, enquanto o último contribui para uma característica descontínua do tipo schumpeteriano. Em processos reais de mudança institucional, todos os três modos podem interagir e brincar nos respectivos papéis em graus variados. Desta forma, Aoki (2005) fazendo uso da teoria dos jogos associa as ideias de Schumpeter à teoria institucionalista. Assim, a inovação sugerida por Schumpeter ocorre no intuito de desenvolver a organização interna de uma empresa, o que faz produzir novos produtos e implantar novas tecnologias para concorrer no mercado, induzindo outras firmas a fazerem o mesmo.

Os formatos organizacionais estão fundamentados no aspecto espacial, bem como na importância das relações entre empresas, e no caso específico dos arranjos produtivos das empresas com outras instituições. Com isso, as pequenas e médias empresas melhoram a competitividade do arranjo produtivo através do aprendizado coletivo e das ações conjuntas, o que permite a redução de suas desvantagens em relação às grandes empresas (CAMPOS e PAULA, 2006).

Os mecanismos de reagregação schumpeteriana: a sobreposição de enraizamento social e as complementaridades dinâmicas institucionais são reais e operam simultaneamente numa forma interativa. O reagrupamento schumpeteriano pode trazer mais inovações aos elementos para o processo de inovação institucional. A evolução do cluster do vale do silício nas duas últimas décadas do século passado é um dos mais extraordinários exemplos da inovação shumpeteriana no sentido institucional (AOKI, 2005).

Neste sentido, com a organização industrial que induz a concorrência e consequentemente leva ao progresso tecnológico, o tamanho das firmas também influência no mercado concorrencial. 


\subsection{Lei de Zipf}

Se existe uma lei empírica que explica a distribuição do tamanho das empresas, provavelmente, há uma proposição de que o tamanho das empresas segue uma lei de potência com expoente próximo a 1. Esta é conhecida por Lei de Zipf em função do trabalho de George Zipf (1949) ${ }^{1}$.

A Lei de Zipf também pode ser chamada pela regra da ordem de tamanho, da qual implica que o produto do faturamento de qualquer empresa multiplicado pela sua posição na ordenação do país será igual ao faturamento da maior empresa. Isto significa dizer que a segunda maior empresa terá a metade do faturamento da maior, a terceira terá o terço, e assim por diante. Desta forma, este trabalho irá abordar dois tipos de pesquisa: a teórica, que busca formular modelos que reproduzam a regra da ordem de tamanho e a empírica, que testa a veracidade da proposição feita por Zipf.

Na literatura sobre o dinamismo de crescimento de negócios existe uma tradição bem estabelecida que descreve a modificação do tamanho das firmas, a um longo período, tal como o efeito cumulativo de diferentes choques originados pelos diversos acidentes que as afetam neste mesmo tempo (KALEKI ,1945).

Desta forma, há algumas tentativas de explicar a Lei de Zipf, que iniciam com a suposição implícita ou explícita de que o conjunto de empresas nasceu em um mesmo período e vivem para sempre. Porém esta abordagem considera que a economia restringe-se a uma única empresa e que a distribuição do tamanho das firmas atinge seu equilíbrio, e ainda se a distribuição do tamanho de uma única empresa atinge um estado estável. Os processos endógenos e exógenos se combinam e formam o tamanho das firmas, que por sua vez espera que sejam parcialmente relevados pelas características da distribuição de empresas. Esta discussão também pode influenciar a criação e destruição de emprego, e ainda o crescimento da produtividade no nível macroeconômico devido ao papel da estrutura de mercado (MALEVERGNE, SAICHEV, SORNETTE, 2009).

A lei de Zipf afirma que, para a maioria dos países, o número de empresas com tamanho maior do que $S$ é inversamente proporcional à $S$. A maioria das explicações começa com a regra de crescimento proporcional, mas a necessidade de Gibrat de integrar as limitações e os ingredientes adicionais introduzem desvios a partir dele. Mostramos que combinando a regra de Gibrat em todos os níveis da empresa com processos aleatórios de nascimentos e mortes das empresas a lei de Zipf funciona como uma condição de "equilíbrio" entre a taxa média de crescimento de empresas já estabelecidas e taxa de crescimento líquido de investimentos em novas empresas entrantes. Assim, a lei de Zipf pode ser interpretada como a condição do longo prazo para a alocação ótima de recursos que garanta o crescimento sustentável da máxima taxa de uma economia. Para as economias com idade finita, a lei de Zipf pode ser recuperada como uma compensação entre o efeito finito-idade e pequenos

${ }^{1}$ É uma lei de potência a qual rege a dimensão, importância dos elementos de uma lista ordenada. Trata-se de sobre a distribuição de valores de acordo com o ranking numa lista. Nesta lista, a empresa $n$ teria uma relação de valor com o primeiro da lista segundo $1 / \mathrm{n}$. Isto significa que a segunda empresa teria a metade do faturamento da primeira. A terceira empresa teria $1 / 3$ e assim sucessivamente. 
desvios da condição de equilíbrio. Assim, é possível explicar que a taxa de risco dependente da presença de um tamanho mínimo abaixo do qual as empresas deixam o mercado. Os resultados não só se mantêm para as médias, mas também se aplicam a uma única economia típica (MALEVERGNE, SAICHEV, SORNETTE, 2009).

$\mathrm{Na}$ literatura sobre a dinâmica de crescimento das firmas, uma tradição bem estabelecida descreve a mudança do tamanho da firma, ao longo de um determinado período de tempo, como efeito cumulativo de um número de diferentes choques originados pelos diversos efeitos que afetaram a empresa neste período. Nesta linha têm-se autores como: Kalecki (1945); Ijri e Simon (1977); Steindl (1965); Sutton (1998) e Geroski (2000).

Este argumento em conjunto com a lei de Gibrat de crescimento proporcional, constitui o ponto de partida para várias tentativas de explicar a lei de Zipf. Contudo, estas tentativas geralmente começam com a suposição implícita ou implícita de que o conjunto das empresas, sob consideração, nasceu na mesma origem do tempo e vivem para sempre. Esta abordagem é equivalente a considerar que a economia é feita de apenas uma única empresa e que a distribuição de tamanho das firmas atinge um estado estacionário, se apenas se a distribuição do tamanho de uma única empresa atinge um estado estacionário. (MALEVERGNE, SAICHEV, SORNETTE, 2009).

Di Patti e Ariccia (2004) e Reynolds et al. (1994) propõem uma abordagem alternativa para modelar uma distribuição de tamanhos das firmas estacionária. É levar em conta o fato das empresas não aparecem todas ao mesmo tempo, mas nasceram de acordo com um fluxo mais ou menos regular de empresas recém criadas.

Simon (1955) foi a primeiro a abordar esta questão. Ele propôs modificar o modelo de Gibrat por representar a entrada de novas empresas ao longo do tempo como a taxa de crescimento total da indústria. Em seguida, ele obteve um estado estacionário da distribuição de tamanhos de empresa com uma cauda superior variando regularmente, cujo expoente $\mathrm{m}$ vai para um no limite de uma infinitamente pequena probabilidade de que uma nova empresa é criada. Krugman (1996) observou que esta situação não é muito relevante para explicar os dados empíricos, na medida em que a convergência em direção ao estado de equilíbrio é, então, infinitamente lento.

Segundo Malevergne, Saichev, Sornette, (2009), autores como Gabaix (1999) permitiu o nascimento de novas firmas, com a probabilidade de criação de uma nova firma de um dado tamanho proporcional ao tamanho das firmas existentes e independente do tempo. $\mathrm{Na}$ verdade, essa suposição não reflete a real dinâmica de criação das empresas. Por exemplo, Bartelsman et al. (2005) argumentou que novas firmas têm um tamanho relativamente pequeno em comparação com o tamanho mais maduro e eficiente das firmas, mas se desenvolvem à medida que crescem. Ou seja, parece irrealista esperar uma probabilidade diferente de zero para o nascimento de uma empresa de tamanho muito grande, digamos, de tamanho comparável à maior atualmente no mercado.

No entanto, o fato de que as empresas podem ir à falência e desaparecer da economia é uma observação fundamental que é muitas vezes negligenciada em modelos. Muitas empresas são conhecidas por sofrer períodos transitórios de decadência que, quando persistentes, podem levar à sua saída do negócio (Di Patti e Ariccia, 2004; Knaup, 2005; Brixy e Grotz, 2007; Bartelsman et al., 2005). Simon (1960), bem como Steindl (1965) têm considerado este fato estilizado dentro de uma generalização de Simon (1955), onde a queda de uma empresa e, 
finalmente, a sua saída ocorre quando o seu tamanho chega a zero. Em Simon (1960) a taxa de saída de empresas compensa exatamente o fluxo de nascimentos das empresas para que a economia atinja o estado estacionário da distribuição de tamanhos.

Em contraste, Steindl (1965) inclui os nascimentos e as mortes, mas dentro de uma indústria com um número crescente de empresas. Um estado de equilíbrio da distribuição é obtido cuja cauda segue uma lei de potência com um expoente que depende da taxa líquida de entrada de novas empresas e da taxa média de crescimento de empresas já estabelecidas. A lei de Zipf apenas é recuperada no limiar onde a taxa de entrada líquida de novas empresas vai para zero. Ambos os modelos contam com a existência de um tamanho mínimo abaixo do qual uma empresa sai do mercado. Esta hipótese corresponde à existência de um mínimo tamanho eficiente, abaixo do qual uma empresa não pode operar, como está bem estabelecida na teoria econômica. No entanto, não pode estar mais de um tamanho mínimo como o nível de saída (morte) de uma empresa e não tem motivos para ser igual ao tamanho de uma firma nova.

A lei de Zipf é representada pela equação:

$\mathrm{LnRi}=\alpha+\beta \mathrm{Ln} \mathrm{Si}$

Onde R é a Posição de cada empresa no Rankig das maiores empresas brasileiras no ano i e $S$ é o faturamento da empresa no ano i. Em termos Absolutos, Se $\beta>1$ tem-se uma distribuição mais equitativa entre o faturamento das maiores empresas. Se $\beta<1$, tem-se uma distribuição mais concentrada; Quando $\beta=1$ tem-se a Lei de Zipf ou lei da ordem de tamanho.

\section{Exposição do Modelo para Dados em Painel}

O modelo de dados para painel permite controlar os efeitos das variáveis não observadas, ou seja, equilibrar as diferenças existentes entre as empresas. Também permite o uso de mais observações, elevando o número de graus de liberdade e reduzindo a colinearidade entre as variáveis explicativas.

Assim, o modelo para os dados em painel é representado por:

$$
y_{i t}=\beta_{0 i t}+\beta_{1 i t} x_{l i t}+\ldots+\beta_{k i t} x_{k i t}+u_{i t}
$$

$\mathrm{Na}$ equação (1), o $i$ denota as diferentes empresas e o $t$ denota o período de tempo que está sendo analisado. $\beta_{0}$ refere-se ao parâmetro de intercepto e $\beta_{k}$ ao coeficiente angular correspondente à k-ésima variável explicativa do modelo. Os $y_{i}$ e $u_{i}$ são vetores de dimensão $(T \times 1)$ e contém, respectivamente, as $T$ rankings (variáveis dependentes) e os $T$ erros. Assim, o elemento $x_{K i t}$ refere-se à k-ésima variável explicativa, o faturamento, para a empresa $i$ no instante de tempo $t$. Finalmente $\beta_{1}$ é o parâmetro a ser estimado.

\subsection{Modelo de Efeitos Fixos}

O modelo de efeitos fixos pode controlar os efeitos das variáveis omitidas que variam entre empresas e continuam constantes ao longo do tempo; e os parâmetros resposta são também constantes para todas as firmas e todos os anos. Segundo Hill et al (2006), as suposições do modelo são: 
O modelo será dado por:

$$
\beta_{0 i t}=\beta_{0 i} \quad \beta_{1 i t}=\beta_{1} \ldots \beta_{k i t}=\beta_{k}
$$

$$
y_{i t}=\alpha_{i}+\beta_{1} x_{1 i t}+\ldots+\beta_{k} x_{k i t}+e_{i t}
$$

A incógnita $\alpha_{i}$ representa os interceptos a serem estimados sendo um para cada firma. Como os parâmetros resposta não variam, as diferenças de comportamento entre as firmas deverão ser captadas pelo intercepto. Desse modo, $\alpha_{i}$ pode ser interpretado como o efeito das variáveis omitidas no modelo.

Para testar se as suposições sobre o modelo estão adequadas é necessário fazer um teste para verificar se os interceptos são diferentes entre as firmas. As hipóteses nula e alternativa seguindo Hill et al. (2006) são:

$$
\begin{aligned}
& H_{0}: \beta_{01}=\beta_{02}=\ldots=\beta_{0 k} \\
& H_{1}: \text { os interceptos } \beta_{0 i} \text { não são todos iguais. }
\end{aligned}
$$

Estas hipóteses podem ser testadas através da estatística $\mathrm{F}$. Rejeitando-se $\mathrm{H}_{0}$, percebese que os interceptos não são todos iguais, satisfazendo a suposição do modelo de $n$ interceptos diferentes.

\subsection{Modelo de Efeitos Aleatórios}

O modelo de efeitos aleatórios possui as mesmas definições do modelo de efeitos fixos, isto é, o intercepto varia de um indivíduo para o outro, mas não ao longo do tempo, e os parâmetros resposta são constantes para todos os indivíduos e em todos os períodos de tempo. A diferença entre os dois modelos está no tratamento do intercepto.

Como abordado anteriormente, o modelo de efeitos fixos expõe os interceptos como parâmetros fixos. Já o de efeitos aleatórios expõe os interceptos como variáveis aleatórias. Ainda de acordo com Hill et al (2006), os n interceptos serão modelados assim:

$$
\beta_{0 i}=\overline{\beta_{0}}+\alpha_{i} \quad i=1, \ldots, n
$$

Nota-se que este intercepto é composto pelo intercepto do modelo de efeitos fixos, $\alpha_{i}$, que capta as diferenças de comportamento das empresas, e por outro componente, $\overline{\beta_{0}}$, que corresponde ao intercepto de conjunto das firmas.

O modelo geral de efeitos fixos é dado por:

$$
y_{i t}=\overline{\beta_{0}}+\beta_{1} x_{1 i t}+\ldots+\beta_{k} x_{k i t}+v_{i t}
$$

Sendo que $v_{i t}=u_{i t}+\alpha_{i}$ representa o erro.

Para testar se o modelo de efeitos aleatórios é apropriado Wooldridge (2010) baseou nas seguintes hipóteses nula e alternativa:

$$
\begin{aligned}
& H_{0}: \sigma_{\alpha}^{2}=0 \\
& H_{1}: \sigma_{\alpha}^{2} \neq 0
\end{aligned}
$$

Wooldridge (2010) defende que o principal determinante na escolha do modelo de efeitos fixos e do modelo de efeitos aleatórios é o efeito não observado $\alpha_{i}$. Há momentos que $\alpha_{i}$ não é correlacionado com todas as variáveis explicativas, o modelo de efeitos variáveis é o mais indicado. Oposto a isto, se $\alpha_{i}$ for correlacionado com algumas variáveis explicativas, o modelo de efeitos fixos deve ser utilizado. 
Ao checar se existe correlação entre $\alpha_{i}$ e as variáveis explicativas, testa-se a hipótese nula, sugerida pelo autor:

$H_{0}=\alpha_{i}$ não é correlacionado com as variáveis explicativas

$H_{1}=\alpha_{i}$ é correlacionado com as variáveis explicativas

A estatística de teste é denominada de teste de Hausman-Taylor proposto por Hausman e Taylor (1981). Caso a hipótese nula seja aceita, não há evidências que $\alpha_{i}$ seja correlacionado com as variáveis explicativas. Sendo assim, o modelo de efeitos aleatórios deve ser utilizado. Por outro lado, se a hipótese alternativa for aceita, deve-se utilizar o modelo de efeitos fixos. ${ }^{2}$

\section{Resultados}

\subsection{Evidências Iniciais}

Evidências iniciais permitem observar o comportamento do percentual das empresas privadas e estatais entre as maiores empresas brasileiras, entre 2003 a 2010, através do Gráfico 01.

Este possui três informações gerais: o primeiro é que o percentual de empresas estatais sempre foi inferior ao percentual das empresas privadas. Porém há momentos em que ambos diminuem, nos anos 2007 e 2008 devido à crise; mas em 2009 o percentual das empresas privadas cresce e das estatais diminuem; e finalmente em 2010 o percentual das privadas decresce e das estatais se elevam por conta, possivelmente, dos incentivos fiscais. O segundo é que há certa tendência na diminuição da participação das estatais que fica em média abaixo dos $20 \%$, só que 2010 ficou em torno de $10 \%$. O percentual das empresas de capital nacional também vem caindo e em 2010 fica em torno de 50\%. E o terceiro é que a parcela do total do faturamento das maiores empresas estatais se mantém em torno de $30 \%$.

GRÁFICO 01: Empresas Privadas e Estatais Brasileiras

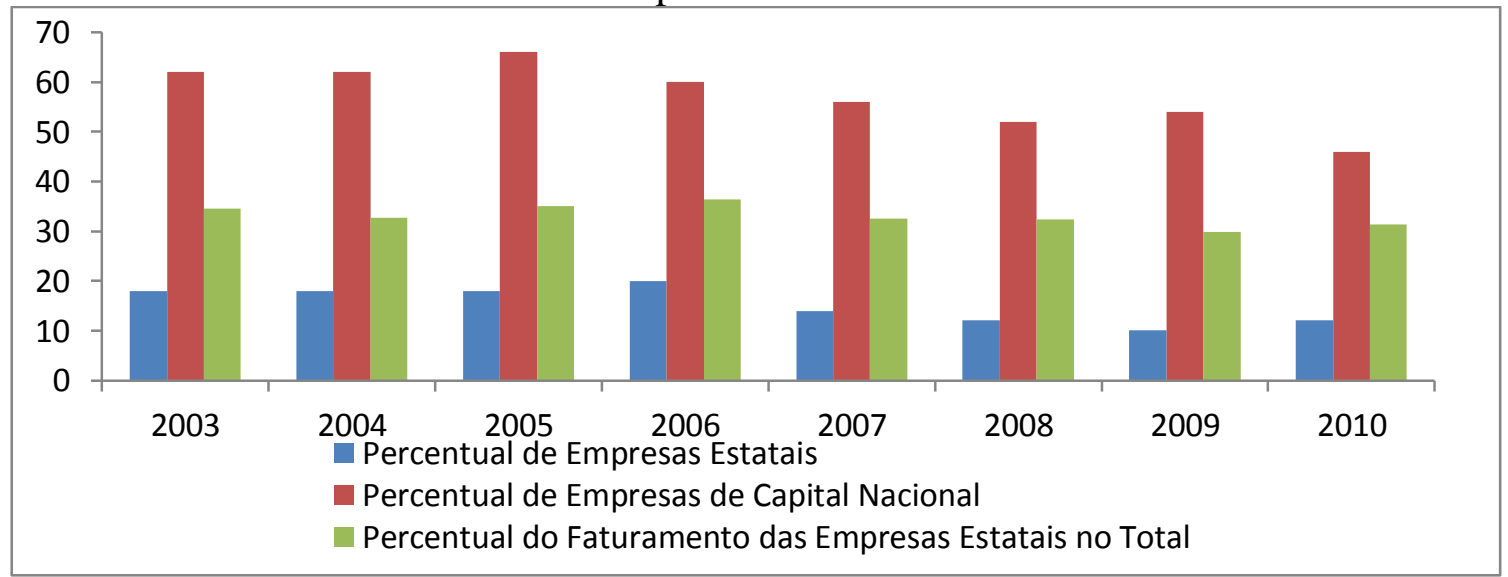

Fonte: Elaborado pelos autores com base no ranking Exame Maiores e Melhores.

\footnotetext{
${ }^{2}$ Os dados foram obtidos do site da Revista Exame, edição especial: "Melhores e maiores empresas - as 500 maiores empresas do Brasil" e, retirados as 50 maiores empresas de 2003 a 2010, e posto em painel, ano a ano. Depois foram subdivididas em 25 maiores e 10 maiores no período de 2003 a 2010. Na estimação do modelo, utilizou-se o software Statal2.
} 
Das 50 maiores empresas, poucas são estatais, e sua quantidade cada vez mais diminui, passando de 9 em 2003 para 6 maiores em 2010; apenas em 2006 possuiu 10 estatais. Nota-se que a Petrobrás sempre atingiu o primeiro lugar no ranking e a Br. Distribuidora o segundo lugar. Porém o faturamento da Petrobrás possuiu o dobro das demais e permanece está estável entre 2003 a 2010 conforme a tabela 01.

Percebe-se ainda que a Cemig que de 2003 a 2005 estava na $3^{\text {a }}$ posição das estatais, caiu para a $4^{\mathrm{a}}$ em 2006; e a Refap subiu de posição e permaneceu na $3^{\mathrm{a}}$ posição de 2006 a 2010. Há outras que antes estavam na lista das 50 maiores em 2003, porém no decorrer dos anos ficaram ausentes, como: Liquigás, Copel Dis. Isto testa aplicabilidade da Lei de Zipf, mencionado anteriormente, que justifica o surgimento e o desaparecimento das empresas estatais no grupo das maiores, podendo ocorrer também nas de capital privado. Este desaparecimento do ranking pode ser justificado pela perca de posição no mercado ou mesmo o fechamento se for o caso.

TABELA 01: Ranking das 5 Maiores Empresas Brasileiras: 2003 a 2010

\begin{tabular}{|c|c|c|c|c|c|c|c|c|}
\hline Rank & 2003 & 2004 & 2005 & 2006 & 2007 & 2008 & 2009 & 2010 \\
\hline 1a & Petrobras & Petrobras & Petrobras & Petrobras & Petrobras & Petrobras & Petrobras & Petrobras \\
\hline $2 \underline{a}$ & BrDistr & BrDistr & BrDistr & BrDistr & BrDistr & BrDistr & BrDistr & BrDistr \\
\hline $3 \underline{a}$ & Cemig & Cemig & Cemig & Refap & Refap & Refap & Refap & Refap \\
\hline $4 \underline{a}$ & Itaipu & ECT & Refap & Cemig & Eletrobr & Eletrob & ECT & ECT \\
\hline $5 \underline{a}$ & Refap & Refap & Ect & ECT & ECT & ECT & Cemig & Cemig \\
\hline
\end{tabular}

Fonte: Elaborado pelos autores com base no ranking Exame Maiores e Melhores.

\subsection{Estatística Descritiva}

As evidências iniciais apontaram para movimentações no ranking das maiores empresas brasileiras no período analisado. Assim, eleva-se a expectativa de aplicabilidade da lei de Zipf.

Na Tabela 02, percebe-se que o faturamento em US\$, tem crescido ao longo dos anos. Contudo, por se tratar de grandes empresas que têm relação com o mercado internacional, estas sentiram o efeito da crise de 2009, pois o faturamento caiu em relação ao ano de 2008 e em 2010 se recuperaram parcialmente em função das políticas econômicas adotadas pelo governo para combater a crise, notadamente a redução de impostos para alguns setores. 
TABELA 02: Estatística Descritiva do Faturamento das 50 Maiores Empresas em Logaritmo

\begin{tabular}{ccccccc}
\hline Ano & Média & $\begin{array}{c}\text { Desvio- } \\
\text { padrão }\end{array}$ & Mínimo & Máximo & Mediana & CV \\
\hline 2003 & 9832.866 & 14113.54 & 3877.3 & 102926.9 & 6284.3 & 1.44 \\
2004 & 10650.37 & 14317.25 & 4287.3 & 104722.1 & 7671.7 & 1.34 \\
2005 & 11003.98 & 16181.33 & 4432.1 & 117176.4 & 7527.4 & 1.47 \\
2006 & 11776.54 & 17984.82 & 4535.5 & 130180.2 & 7659.1 & 1.53 \\
2007 & 12645.85 & 18019.93 & 4936.6 & 130408.9 & 8443.85 & 1.42 \\
2008 & 13538.76 & 19822.82 & 5253.3 & 143189.5 & 9222.00 & 1.46 \\
2009 & 11887.07 & 15939.86 & 4479.4 & 113809.1 & 8489.1 & 1.34 \\
2010 & 12968.67 & 17944.43 & 5275.9 & 126339.8 & 8731.35 & 1.38
\end{tabular}

Fonte: Elaborado pelos autores com base no ranking Exame Maiores e Melhores.

Em geral observa-se que há uma tendência de elevação da média, mediana, faturamento da maior empresa e faturamento da menor empresa no ranking considerado até 2008. Em 2009 há uma queda e em 2010 em virtude vê-se uma leve recuperação.

O reflexo da crise de 2008 repercutiu diferentemente entre as empresas. Enquanto a maior empresa a queda no faturamento entre 2008 e 2009 foi de $20,52 \%$ para a menor a queda foi de $14,73 \%$. Já para a média a queda foi de $12,20 \%$. Em parte este comportamento reflete o efeito da apreciação cambial e o peso do dólar na receita da empresa. Há empresas com maior relação com o mercado internacional seja na compra de matérias primas seja na exportação de produtos.

Da mesma forma que as ações das políticas de incentivo repercutem diferentemente entre as empresas assim como outras medidas estratégicas adotadas pelas próprias empresas. A Petrobrás que é a maior empresa apresenta um aumento de $11,01 \%$ entre 2009 e 2010 . Para a menor empresa a elevação foi de $17,78 \%$. Já para a média das empresas foi de $9,10 \%$.

Já em relação à dispersão medida pelo coeficiente de variação $(\mathrm{CV})$ não se tem uma tendência definida, pois diminuiu entre alguns anos mais volta a subir em outros. $\mathrm{O}$ ano de menor dispersão é 2009. Isto ocorre justamente em virtude da maior empresa do ranking ser mais afetada que a média do setor. De fato isto é corroborado pela mediana. Em 2009, tem-se o maior valor pelo período analisado.

Na Tabela 03, ressalta-se que se Ln S > 0 significa que a curva que relaciona a ordem e o tamanho das firmas é convexa, e as maiores firmas possuem maior influência na economia e as menores possuem pouca influência, contrariando o que prevê a regra da ordem de tamanho das firmas. Se Ln S é positivo e crescente quer dizer que a evolução temporal do tamanho das empresas está positivamente correlacionada com sua dimensão, gerando um crescimento divergente em que as maiores empresas crescem mais do que as menores. Por outro lado, se Ln $\mathrm{S}<0$, o crescimento é convergente e as menores empresas crescem mais do que as maiores. 
Todos os coeficientes são significantes a $1 \%$ e os sinais da variável LNS apresentamse de acordo com o esperado.

Os valores do coeficiente da variável Ln $\mathrm{S}$ estão a depender da quantidade de empresas colocadas no painel. A lei de Zipf se aplica com o coeficiente da variável LNS ficando próximo da unidade em termos absolutos quando o painel é composto pelas dez maiores empresas. À medida que se eleva o número de empresas o coeficiente da variável Ln $\mathrm{S}$ se eleva em valores absolutos apontando para uma convergência, ou seja, as menores empresas estariam crescendo mais rapidamente que as maiores ao longo do período. Assim estaria em discordância com a lei de Zipf. O teste de Hausman sugere que o modelo de efeitos fixos é o mais adequado.

TABELA 03: Lei de Zipf para as Maiores Empresas Brasileiras

\begin{tabular}{lcccccc}
\hline \multicolumn{7}{c}{ Variável Dependente: Ln R } \\
\hline Variável & Modelo & Modelo & Modelo & Modelo & Modelo & Modelo \\
& $(1)$ & $(2)$ & $(3)$ & $(4)$ & $(5)$ & $(6)$ \\
\hline Constante & $16,379^{*}$ & $16,019^{*}$ & $15,055^{*}$ & $14,748^{*}$ & $11,413^{*}$ & $11,118^{*}$ \\
& $(0,173)$ & $(0,201)$ & $(0,326)$ & $(0,346)$ & $(0,477)$ & $(0,487)$ \\
LN S & $-1,474^{*}$ & $-1,435^{*}$ & $-1,342^{*}$ & $-1,309^{*}$ & $-0,994^{*}$ & $-0,964^{*}$ \\
& $(0,019)$ & $(0,022)$ & $(0,034)$ & $(0,036)$ & $(0,048)$ & $(0,049)$ \\
\hline Teste F & 6023,73 & 4220,58 & 1523,61 & 1292,65 & 432,49 & 390,14 \\
Teste de & & & & & & \\
Hausman & $-77,61$ & $-64,97$ & $-39,03$ & $-35,95$ & $-20,80$ & $-19,75$ \\
N. Obs & 400 & 400 & 200 & 200 & 80 & 80 \\
\hline
\end{tabular}

Fonte: Elaborado pelos autores com base no ranking Exame Maiores e Melhores.

Nota: Desvio-padrão entre parênteses. * significante a $1 \%$.

O Gráfico 2 traz os valores dos coeficientes da variável Ln S estimados ao longo dos anos para os três grupos. Um grupo que inclui as 50 maiores empresas, um segundo que inclui as 25 maiores e finalmente o último que inclui apenas as 10 maiores empresas. Para o grupo das 10 maiores empresas a regularidade da Ordem do Tamanho das empresas é verificada, pois o coeficiente se mantém em torno da unidade ao longo dos anos.

GRÁFICO 02: Coeficiente de Pareto para os Grupos de Empresas

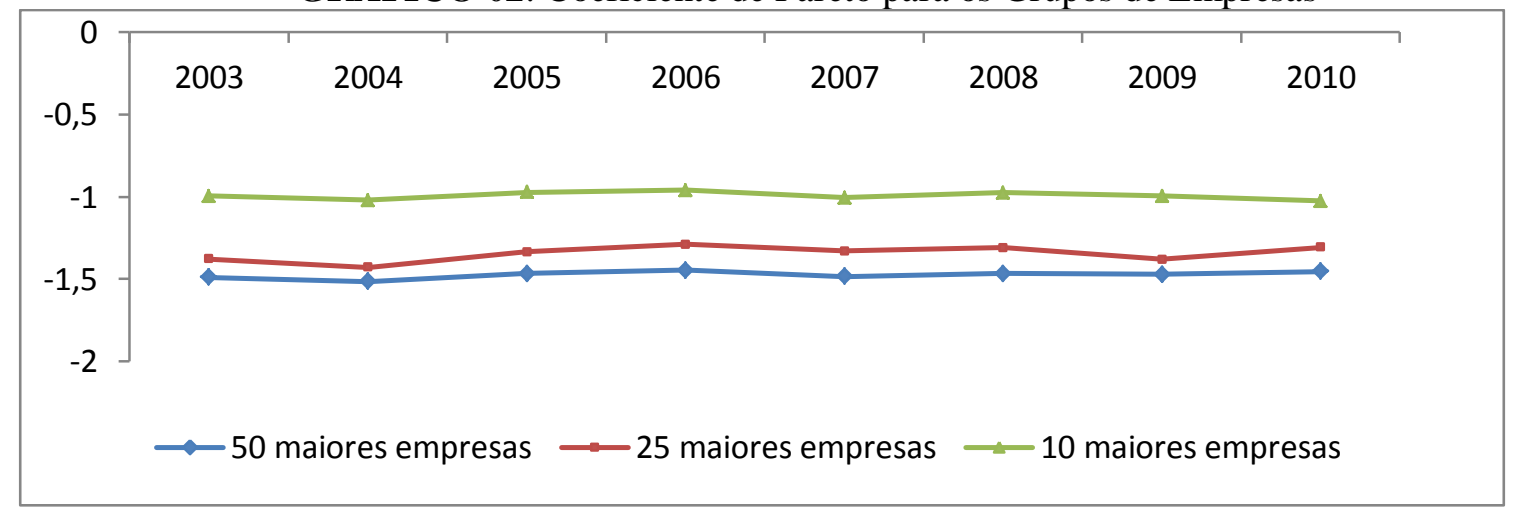

Fonte: Elaborado pelos autores com base no ranking Exame Maiores e Melhores. 


\section{CONCLUSÕES}

A teoria da distribuição dos tamanhos das empresas foi explorada em outros países e pouco explorada no Brasil. A ideia é verificar se a hipótese da Lei de Zipf se aplica para as maiores empresas brasileiras após o desenvolvimento de teorias em várias áreas do conhecimento e em particular na organização industrial para esta regularidade empírica.

Trabalhou-se com o ranking das maiores empresas brasileiras e dividiu-se a amostra em três grupos. Os dados foram organizados em painel considerando o período de 2003 a 2010 que coincide com os dois mantados do Presidente Lula.

Inicialmente pode-se perceber que o percentual de empresas estatais sempre foi inferior às empresas privadas; o percentual das empresas de capital nacional também vem caindo e em 2010 fica em torno de 50\%; e a parcela do total do faturamento das maiores empresas pelas empresas estatais se mantém em torno de $30 \%$.

Das 50 maiores empresas poucas são estatais, e sua quantidade cada vez mais diminui, de 9 em 2003 para 6 maiores em 2010. Nota-se que a Petrobrás sempre ocupou o primeiro lugar no ranking e a $\mathrm{Br}$. Distribuidora o segundo lugar.

Há movimentações de entrada e saída de outras firmas na lista das 50 maiores em 2003. Porém, no decorrer dos anos ficaram ausentes, como: Liquigás, Copel Dis. Isto testa aplicabilidade da Lei de Zipf, mencionado anteriormente, que justifica o surgimento e o desaparecimento das empresas estatais no ranking, podendo ocorrer também nas de capital privado. Este desaparecimento do ranking pode ser justificado pela perda de posição no mercado ou mesmo o fechamento se for o caso e até mesmo fusões.

Já em relação à dispersão entre o faturamento das empresas medida pelo coeficiente de variação $(\mathrm{CV})$ não se tem uma tendência definida, pois diminuiu entre alguns anos mais volta a subir em outros. O ano de menor dispersão é 2009. Isto ocorre justamente em virtude da maior empresa ser mais afetada que a média do setor com a crise de 2009. De fato isto é corroborado pela mediana. Em 2009, tem-se o maior valor pelo período analisado.

O efeito e a recuperação das empresas com a crise de 2008 ocorrem de forma diferente quando se olha para a empresa de maior faturamento, menor e o faturamento médio das empresas. Em parte isto reflete o efeito das políticas de incentivo adotadas pelo governo para enfrentamento da crise e em parte pelo grau de exposição à economia externa de cada empresa.

Os resultados da estimação da equação da Lei de Zipf mostram que o crescimento do tamanho das empresas é convergente quando são agrupadas as 50 maiores empresas indicando que as menores empresas do ranking apresentam taxas de crescimento do faturamento maior que as empresas que lideram o grupo. Considerando apenas as 25 maiores empresas esta convergência diminui. E, finalmente considerando apenas as 10 maiores empresas esta convergência desaparece. Portanto, a hipótese da Lei de Zipf somente se aplica quando são agrupadas apenas as 10 maiores empresas. 


\section{REFERÊNCIAS}

AOKI, Masahiko. Schumpeterian Innovation of Instituion. Tenth Conference of the International Schumpeter Society held in Milan on January, 2005.

BARTELSMAN, E., SCARPETTA, S., SCHIVARDI, F. Comparative Analysis of Firm Demographics and Survival: Micro-level Evidence for the OECD Countries, Industrial and Corporate Changes 14, 365-391, 2005.

BRIXY, U. and GROTZ, R. Regional patterns and determinants of birth and survival of new firms in Western Germany, Entrepreneurship \& Regional Development 19 (4), p. 293-312, 2007.

CAMPOS, Antonio Carlos de; e PAULA, Nilson Maciel de. Novas formas de organização industrial e o conceito de firma: uma abordagem neo-schumpeteriana. Ensaios FEE, Porto Alegre, v. 27, $\mathrm{n}^{\circ} 1$, p.31-56, maio de 2006.

CANO, Wilson. América Latina: notas sobre a crise atual. Econ. Soc. Vol. 18 n³ Campinas, dezembro de 2009.

CANO, Wilson; e SILVA, Ana Lucia Gonçalves da. Política industrial do governo Lula. Texto para discussão. IE/UNICAMP nº 181, julho de 2010.

DI PATTI, Bonaccorsi, E. and ARICCIA, G. Dell'. Bank competition and firm creation. Journal of Money Credit and Banking 36, p. 225-251, 2004.

DUARTE, P.C.; LAMOUNIER, W. M.; TAKAMATSU, Renata Turola. Modelos econométricos para dados em painel: aspectos teóricos e exemplos de aplicação à pesquisa em contabilidade e finanças. (2007) Acesso em: 27 de novembro de 2012. Disponível em: <www.congressousp.fipecafi.org/artigos72007/523.pdf>

GABAIX, X. Zipf's Law for Cities: An Explanation. Quarterly Journal of Economics 114, p.739-767, 1999.

GEROSKI, P. The Growth of Firms in Theory and in Practice, in N. Foss and V. Malinke (eds.), New Directions in Economic Strategy Research. Oxford University Press, 2000.

HILL, R. C.,GRIFFITHS, W.E., JUDEGE, G.G. Econometria. São Paulo: Saraiva, 2006.

HAUSMAN, J. A., and TAYLOR, W. E. Panel Data and Unobservable Individual Efects. Econometrica, 49, p. 1377-1398, 1981.

IJRI, Y. and SIMON, H. A. Skew Distributions and the Sizes of Business Firms. NorthHolland, New

York, 1977.

JUSTO, Wellington Ribeiro. Um Século de Crescimento das Cidades Brasileiras: Uma abordagem espacial (1910-2010). In: Anais do 40 Encontro Nacional de Economia. ANPEC, Ipojuca, 2012.

JUSTO,W.R. Zipf's Law and the Gibrat's Law: What Do the Facts Have to Say about the Brazilian Cities? Journal of Finance and Economics, Vol. 2, No. 5, 136-144, 2014. 
KALECKI, M. On the Gibrat Distribution. Econometrica 13, p. 161-170, 1945

KNAUP, A. E. Survival and longevity in the Business Employment Dynamics data. Monthly Labor Review 128 (5): 50-56, 2005.

MALEVERGNE, Y; SAICHEV, A; SORNETTE, D. Zipf's law for firms: relevance of birth and death processes. (2009).

REYNOLDS, P.D., STOREY D.J. WESHEAD and P. Cross-national comparisons of the variation in new firm formation rates. Regional Studies 28, p. 443-456, 1994.

SIMON, H. A. On a class of skew distribution functions, Biometrika 42, p. 425-440, 1955.

SIMON, H. A. Some further notes on a class of skew distribution functions. Information and Control 3, p. 80-88, 1960.

STEINDL, J. Random processes and the growth of firms. A study of the Pareto law. London: Charles Griffin \& Company), 1965.

SUTTON, J. Gibrat's legacy, Journal of Economic Literature 35, p, 40-59, 1997.

ZIPF, G.K. Human Behavior and the Principle of Least-Effort. Addison-Wesley, Cambridge, MA, 1949.

WOOLDRIDGE, M. J. Econometric Analysis of Cross Section and Panel Data. Second Edition. The MIT Press. Cambridge, Massachusetts, London, England, 1096p. 2010. 